Revista Brasil. Bot., V.25, n.2, p.215-220, jun. 2002

\title{
Síndromes de dispersão de espécies arbustivo-arbóreas em cerrado sensu stricto do Brasil Central e savanas amazônicas
}

\author{
DANIEL L.M. VIEIRA ${ }^{1}$, FABIANA G. AQUINO ${ }^{2,4}$, MÁRCIA A. BRITO', \\ CLARISSA FERNANDES-BULHÃO ${ }^{3}$ e RAIMUNDO P. B. HENRIQUES ${ }^{1}$
}

(recebido: 6 de setembro de 2000; aceito: 20 de março de 2002)

\begin{abstract}
Dispersal syndromes of shrub-woody species in the cerrado sensu stricto vegetation of Central Brazil and amazonian savannas). The objective was to identify the proportion of seed dispersion syndromes in areas of cerrado sensu stricto of Central Brazil and amazonian savannas. Lists of species of nine cerrado and five amazonian savanna areas were used. Dispersion syndromes were determined through literature data. Chi-square test $\left(\chi^{2}\right)$ was used to evaluate possible differences in proportion of zoocory and anemocory among cerrado sensu stricto of Central Brazil and among amazonian savanna areas. Floristic similarity was determined among the 14 areas. The averages of these proportions were compared through $\mathrm{t}$ test and no significant differences were found $(\mathrm{p}>0.05)$. The proportion of zoocory species in cerrado sensu stricto and amazonian savannas were $56.7 \% \pm 5.4 \mathrm{DP}$ and $55.6 \% \pm 11.7 \mathrm{DP}$; the proportion of anemocory were $39.7 \% \pm 6.2 \mathrm{DP}$ and $44.4 \% \pm 11.7 \mathrm{DP}$, respectively. In the cerrado sensu stricto of Central Brazil the proportion of autocory ranged from 1.4 to $5 \%$, while the amazonian savannas presented no autocoric species. Autocory is a rarely found strategy in cerrado sensu stricto and its absence in amazonian savanna areas could be related to the low efficiency in colonization of isolated areas.
\end{abstract}

RESUMO - (Síndromes de dispersão de espécies arbustivo-arbóreas em cerrados sensu stricto do Brasil Central e savanas amazônicas). O objetivo deste trabalho foi identificar as proporções das síndromes de dispersão de sementes em áreas de cerrado sensu stricto do Brasil Central, comparando-as com as de áreas isoladas de savanas amazônicas. Foram utilizadas listas de espécies de nove áreas de cerrado e cinco áreas de savanas amazônicas. Determinou-se a similaridade florística entre as áreas. As síndromes de dispersão de espécies ocorrentes nestas áreas foram determinadas a partir de dados da literatura. Utilizou-se o teste Qui-quadrado $\left(\chi^{2}\right)$ a fim de detectar possíveis diferenças nas proporções de zoocoria e anemocoria entre as áreas de cerrado e entre as áreas de savanas amazônicas. As médias entre as duas regiões foram comparadas pelo teste t. Não foram encontradas diferenças significativas para as duas análises $(\mathrm{p}>0,05)$. A média das proporções de zoocoria para o cerrado sensu stricto foi de 56,7\% $\pm 5,4$ DP e para as savanas amazônicas 55,6\% $\pm 11,7$ DP, e a média das proporções de anemocoria no cerrado sensu stricto foi de 39,7\% $\pm 6,2 \mathrm{DP}$ e para as savanas amazônicas 44,4\% $\pm 11,7$ DP. No cerrado sensu stricto, a autocoria variou de 1,4 a $5 \%$, porém não se verificou a presença de espécies autocóricas nas savanas amazônicas. A autocoria é uma estratégia de dispersão raramente encontrada no cerrado e sua ausência nas áreas de savanas amazônicas pode estar relacionada à sua baixa eficiência em colonizar áreas isoladas.

Key words - Seed dispersal syndromes, cerrado sensu stricto, Central Brazil, amazonian savannas

\section{Introdução}

O cerrado estende-se por uma grande área contínua no Brasil Central e ocorre, também, em áreas descontínuas ao sul e ao norte do país (Ribeiro \& Walter 1998). As manchas disjuntas de cerrado dispersas na floresta úmida nos Estados do Amapá, Amazonas, Pará e Roraima são conhecidas como savanas amazônicas (Prance 1996).

\footnotetext{
1. Universidade de Brasília, Departamento de Ecologia, Caixa Postal 04631, 70910-900 Brasília, DF, Brasil.

2. Embrapa Cerrados, Caixa Postal 08223, 73301-970 Planaltina, DF, Brasil.

3. Universidade de Brasília, Departamento de Botânica, Caixa Postal 04457, 70910-900 Brasília, DF, Brasil.

4. Autor para correspondência: fabiana@cpac.embrapa.br
}

As savanas amazônicas apresentam menos endemismo e diversidade de espécies em relação ao cerrado contínuo, porém existem espécies compartilhadas por estas duas regiões (Prance 1987, Ratter et al. 1996). Poucos estudos foram realizados na tentativa de entender a distribuição das espécies nas savanas amazônicas, bem como as características destas comunidades (Gottsberger \& Morawetz 1986, Miranda 1993, Prance 1996). Estas áreas, por estarem isoladas da distribuição contínua do cerrado, podem apresentar limitações na colonização de espécies, devido à sua grande distância da fonte de propágulos. Um dos fatores que influencia na colonização de habitats é o mecanismo de dispersão das espécies (van der Pijl 1982).

Alguns estudos realizados sobre a dispersão de sementes de plantas no cerrado sensu stricto mostraram que a maioria destas espécies é zoocórica (Gottsberger 
\& Silberbauer-Gottsberger 1983, Batalha \& Mantovani 2000). Os dados encontrados por Oliveira \& Moreira (1992), em um cerrado no Brasil Central, sugerem que a anemocoria é mais comum em fisionomias abertas do que em formações florestais, confirmando a afirmação de Howe \& Smallwood (1982) de que a falta de um dossel contínuo favorece as espécies com dispersão pelo vento.

Embora existam trabalhos pontuais sobre síndrome de dispersão no cerrado sensu stricto do Brasil Central e savanas amazônicas, não se realizou, até o momento, nenhuma comparação deste aspecto entre estas regiões. Sendo assim, os objetivos deste trabalho foram: determinar as proporções das síndromes de dispersão das espécies nas áreas de cerrado sensu stricto (s.str.) do Brasil Central e nas áreas de savanas amazônicas e comparar as proporções das síndromes de dispersão das espécies entre estas regiões.

\section{Material e métodos}

Foram utilizadas listagens de plantas retiradas de estudos realizados em nove áreas de cerrado (s.str.), no Brasil Central (Ratter et al. 1973, Felfili et al. 1993, Álvares-da-Silva 1996), e cinco áreas de savanas amazônicas (Miranda 1993, Sanaiotti 1996) (figura 1). O método de amostragem e o tamanho de cada área, bem como demais informações são apresentadas na tabela 1 .

O cerrado do Brasil Central caracteriza-se por uma forte sazonalidade, com a presença de uma estação seca e outra chuvosa bem definidas. O clima é classificado como AW de Köppen (tropical chuvoso), com média anual de precipitação de $1.500 \mathrm{~mm}$ (Ribeiro \& Walter 1998). Nas savanas amazônicas, o clima é classificado como tropical quente subúmido, em Roraima e equatorial quente úmido, no Amapá, Alter do Chão e Humaitá. A pluviosidade varia entre 1.500 e $2.750 \mathrm{~mm}$ anuais (Sanaiotti 1996).

Utilizou-se a lista de espécies apresentada por Mendonça et al. (1998) para verificar se as espécies presentes nas listagens florísticas das savanas amazônicas se encontravam na região do cerrado. Para determinar a similaridade florística entre as áreas utilizou-se o Índice de Similaridade (S) (Odum 1988):

$$
\mathrm{S}=\frac{2 \mathrm{C}}{\mathrm{A}+\mathrm{B}}
$$

onde: $\mathrm{A}=$ número de espécies na amostra $\mathrm{A}$;

$\mathrm{B}=$ número de espécies na amostra $\mathrm{B}$;

$\mathrm{C}=$ número de espécies comuns nas duas amostras.

As síndromes de dispersão seguiram a classificação de van der Pijl (1982). Este autor classificou as espécies em três grandes categorias: zoocórica - espécie dispersa por animais; anemocórica - espécie dispersa pelo vento;

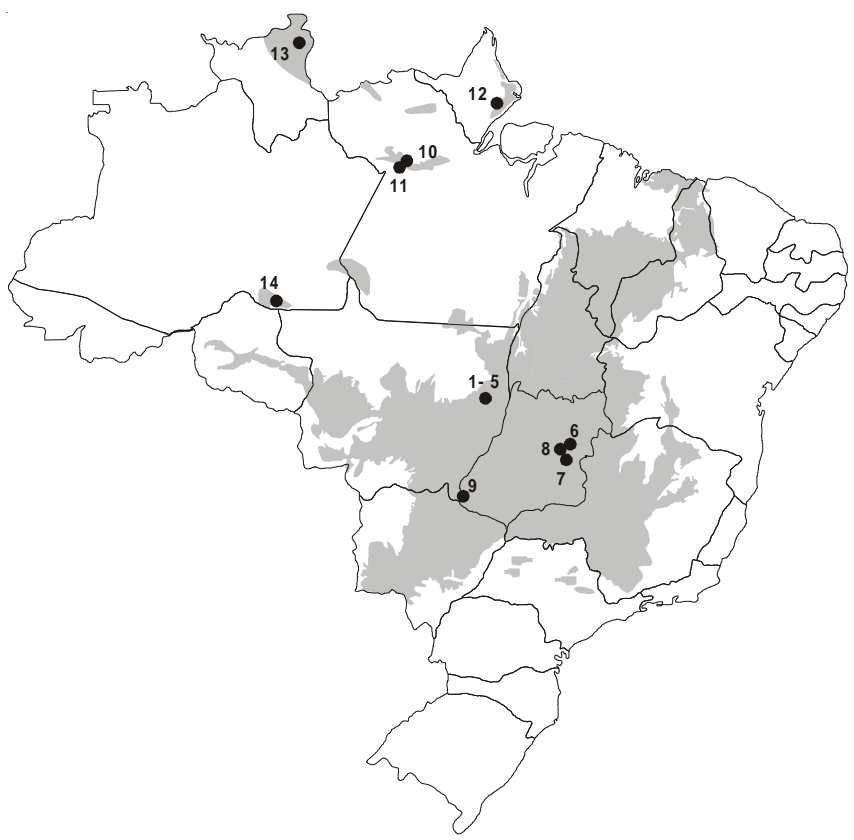

Figura 1. Áreas utilizadas no presente estudo. Cerrado sensu stricto do Brasil Central: 1-5. Nova Xavantina/Cachimbo (MT-1 a MT-5); 6. Estação Ecológica de Águas Emendadas (EEAE/DF); 7. Área de Proteção Ambiental Gama-Cabeça do Veado (APA/DF); 8. Parque Nacional de Brasília (PNB/ DF) e 9. Parque Nacional de Emas (EMAS /GO). Savanas amazônicas: 10. Alter do Chão 1; 11. Alter do Chão 2; 12. Amapá; 13. Roraima e 14. Humaitá. Em cinza: distribuição do Bioma Cerrado.

autocórica - auto-dispersão. As síndromes de dispersão das espécies das áreas de estudo foram determinadas de acordo com os trabalhos de Gottsberger \& Silberbauer-Gottsberger (1983), Miranda (1991), Oliveira \& Moreira (1992), van Roosmalen (1985) e as observações de campo dos autores.

Foram incluídas no estudo apenas plantas identificadas ao nível de espécie e gênero. Nesta última categoria, a determinação da síndrome foi baseada em Gentry (1982). Duas espécies presentes na listagem de Humaitá e duas presentes no Parque Nacional de Emas foram excluídas da análise, pois não foram encontrados dados sobre os seus mecanismos de dispersão.

Para detectar diferenças na proporção de síndromes de dispersão entre áreas de cerrado (s.str.) e, também, entre áreas das savanas amazônicas estudadas, foi utilizado o teste Qui-quadrado $\left(\chi^{2}\right)$ (Zar 1999). As médias das proporções de zoocoria e anemocoria entre o cerrado (s.str.) e as savanas amazônicas foram comparadas pelo teste $\mathrm{t}$ (Zar 1999).

\section{Resultado}

No total, 177 espécies estavam presentes nas listagens das áreas de cerrado (s.str.) do Brasil Central 
Tabela 1. Áreas utilizadas para o levantamento das síndromes de dispersão: cerrado sensu stricto do Brasil Central: Nova Xavantina/Cachimbo (MT-1 a MT-5)*; Estação Ecológica de Águas Emendadas (EEAE/DF); Área de Proteção Ambiental Gama-Cabeça do Veado (APA/DF); Parque Nacional de Brasília (PNB/DF) e Parque Nacional de Emas (EMAS /GO). Savanas amazônicas: Alter do Chão 1; Alter do Chão 2; Amapá; Roraima e Humaitá. S - Número total de espécies. Síndromes de Dispersão: Zoo - Zoocórica; Ane - Anemocórica; Aut - Autocórica.

\begin{tabular}{|c|c|c|c|c|c|c|c|c|}
\hline \multirow[b]{2}{*}{ Áreas } & \multirow[b]{2}{*}{ Coordenadas geográficas } & \multirow{2}{*}{$\begin{array}{l}\text { Métodos de } \\
\text { amostragem }\end{array}$} & \multirow{2}{*}{$\begin{array}{l}\text { Área amostr: } \\
\text { (ha) }\end{array}$} & \multicolumn{4}{|c|}{ Síndromes } & \multirow[b]{2}{*}{ Referências } \\
\hline & & & & $\mathrm{S}$ & $\overline{Z o o}$ & Ane & $\overline{\text { Aut }}$ & \\
\hline \multicolumn{9}{|c|}{ Cerrrado sensu strictu } \\
\hline MT-1 & $12^{\circ} 49^{\prime} \mathrm{S}, 51^{\circ} 46^{\prime} \mathrm{W}$ & Parcelas & 0,08 & 45 & 23 & 21 & 01 & Ratter et al. (1973) \\
\hline MT-2 & $12^{\circ} 49^{\prime} \mathrm{S}, 51^{\circ} 46^{\prime} \mathrm{W}$ & Parcelas & 0,06 & 49 & 27 & 20 & 02 & Ratter et al. (1973) \\
\hline MT-3 & $12^{\circ} 49^{\prime} \mathrm{S}, 51^{\circ} 46^{\prime} \mathrm{W}$ & Parcelas & 0,08 & 62 & 38 & 22 & 02 & Ratter et al. (1973) \\
\hline MT-4 & $12^{\circ} 49^{\prime} \mathrm{S}, 51^{\circ} 46^{\prime} \mathrm{W}$ & Parcelas & 0,22 & 39 & 23 & 14 & 02 & Ratter et al. (1973) \\
\hline MT-5 & $12^{\circ} 49^{\prime} \mathrm{S}, 51^{\circ} 46^{\prime} \mathrm{W}$ & Parcelas & 0,02 & 52 & 27 & 22 & 03 & Ratter et al. (1973) \\
\hline EEAE/DF & $15^{\circ} 31^{\prime}$ a $15^{\circ} 35^{\prime} \mathrm{S}, 47^{\circ} 32^{\prime}$ a $47^{\circ} 37^{\prime} \mathrm{W}$ & V Parcelas & 1,00 & 71 & 38 & 32 & 01 & Felfili et al. (1993) \\
\hline APA/DF & $15^{\circ} 52^{\prime}$ a $15^{\circ} 59^{\prime} \mathrm{S}, 47^{\circ} 50^{\prime}$ a $47^{\circ} 58^{\prime} \mathrm{W}$ & V Parcelas & 1,00 & 64 & 35 & 28 & 01 & Felfili et al. (1993) \\
\hline $\mathrm{PNB} / \mathrm{DF}$ & $15^{\circ} 37^{\prime}$ a $15^{\circ} 45^{\prime} \mathrm{S}, 47^{\circ} 54^{\prime}$ a $47^{\circ} 59^{\prime} \mathrm{W}$ & V Parcelas & 1,00 & 56 & 31 & 23 & 02 & Felfili et al. (1993) \\
\hline EMAS/GO & $17^{\circ} 49^{\prime}$ a $18^{\circ} 28^{\prime} \mathrm{S}, 52^{\circ} 39^{\prime}$ a $53^{\circ} 10^{\prime} \mathrm{W}$ & V Parcelas & 0,48 & $62 * *$ & $* 41$ & 16 & 03 & Álvares-da-Silva (1996) \\
\hline \multicolumn{9}{|l|}{ Savanas amazônicas } \\
\hline Alter do Chão 1 & $02^{\circ} 27^{\prime} \mathrm{S}, 54^{\circ} 50^{\prime} \mathrm{W}$ & Quadrantes & 2,25 & 19 & 08 & 11 & - & Miranda (1993) \\
\hline Alter do Chão 2 & $02^{\circ} 31^{\prime} \mathrm{S}, 54^{\circ} 56^{\prime} \mathrm{W}$ & Quadrantes & 0,96 & 16 & 07 & 09 & - & Sanaiotti (1996) \\
\hline Amapá & $00^{\circ} 27^{\prime} \mathrm{N}, 51^{\circ} 05^{\prime} \mathrm{W}$ & Quadrantes & 2,47 & 12 & 08 & 04 & - & Sanaiotti (1996) \\
\hline Roraima & $04^{\circ} 53^{\prime} \mathrm{N}, 60^{\circ} 54^{\prime} \mathrm{W}$ & Quadrantes & 2,33 & 08 & 05 & 03 & - & Sanaiotti (1996) \\
\hline Humaitá & $08^{\circ} 30^{\prime} \mathrm{S}, 61^{\circ} 38^{\prime} \mathrm{W}$ & Quadrantes & 1,14 & $29 * *$ & $* 17$ & 10 & - & Sanaiotti (1996) \\
\hline
\end{tabular}

* As áreas de cerrado MT1, MT2, MT3, MT4, MT5 correspondem às estações 6, 7, 8, 9, 10, respectivamente, do trabalho de Ratter et al. (1973).

** Foram excluídas duas espécies de cada uma destas áreas, pois não foram encontradas informações sobre suas síndromes de dispersão.

e 48 espécies estavam presentes naquelas das savanas amazônicas. Das espécies presentes nas savanas amazônicas estudadas, apenas uma, Anacardium gigateum Hancock (Anacardiaceae), não ocorreu na região do cerrado, de acordo com a listagem florística de Mendonça et al. (1998).

A similaridade florística foi maior entre as áreas de cerrado da APA Gama-Cabeça do Veado/DF e do Parque Nacional de Brasília/DF $(0,83)$ e menor entre o cerrado da Estação Ecológica de Águas Emendadas/ DF e a savana amazônica de Roraima $(0,03)$ (tabela 2$)$.

Entre as áreas de cerrado (s.s.), a proporção de espécies zoocóricas variou de 51,1 a $68,3 \%$ e a de espécies anemocóricas, de 26,7 a 46,7\% (figura 2). Não houve diferença significativa na proporção das síndromes de dispersão entre as áreas de cerrado (s.s.) $\left(\chi^{2}=6,73 ;\right.$ g.l. $\left.=8 ; p=0,57\right)$. Nas savanas amazônicas, a proporção de espécies zoocóricas variou entre 42,1 e $66,7 \%$, e de anemocóricas entre 33,3 e 57,9\% (figura 2). Da mesma forma, não se encontrou diferença significativa na proporção das síndromes entre as áreas de savanas amazônicas $\left(\chi^{2}=3,63\right.$; g.1. $\left.=4 ; \mathrm{p}=0,46\right)$. No cerrado (s.s), a autocoria variou de 1,4 a $5 \%$, e não se verificou a presença de espécies autocóricas nas savanas amazônicas (figura 2).

A média das proporções de zoocoria para o cerrado (s.s.) foi de $56,7 \% \pm 5,4$ DP e para as savanas amazônicas 55,6\% \pm 11,7 DP. Não houve diferença significativa entre as regiões para esta síndrome $(\mathrm{t}=0,24 ; \mathrm{g} .1 .=12 ; \mathrm{p}=0,81)$. A média das proporções de anemocoria no cerrado (s. str.) foi de $39,7 \% \pm 6,2$ DP e para as savanas amazônicas $44,4 \% \pm 11,7$ DP. Da mesma forma, não houve diferença significativa entre as regiões $(\mathrm{t}=-0,99 ; \mathrm{g} .1 .=12 ; \mathrm{p}=0,34)$ (figura 3 ).

\section{Discussão}

Os valores de similaridade florística, no geral, foram baixos, indicando que o bioma cerrado apresenta alta heterogeneidade florística. Ratter et al. (2000) mostraram grandes diferenças na composição florística comparando 316 sítios de cerrado em vários estados brasileiros. A maior similaridade florística entre as áreas de cerrado da APA Gama-Cabeça do Veado e do Parque Nacional de Brasília e entre as áreas de cerrado de Nova Xavantina em Mato Grosso, provavelmente foi devida 
Tabela 2. Similaridade florística entre as 14 áreas utilizadas para o levantamento das síndromes de dispersão. Cerrado sensu stricto do Brasil Central: 1-5. Nova Xavantina/Cachimbo (MT1 a MT5); 6. Estação Ecológica de Águas Emendadas/DF (EEAE/DF); 7. Área de Proteção Ambiental Gama-Cabeça do Veado/DF (APA/DF); 8. Parque Nacional de Brasília/DF (PNB/DF) e 9. Parque Nacional de Emas/GO (EMAS/GO). Savanas amazônicas: 10. Alter do Chão 1; 11. Alter do Chão 2; 12. Amapá; 13. Roraima e 14. Humaitá.

\begin{tabular}{|c|c|c|c|c|c|c|c|c|c|c|c|c|c|}
\hline & 1 & 2 & 3 & 4 & 5 & 6 & 7 & 8 & 9 & 10 & 11 & 12 & 13 \\
\hline 1 & & & & & & & & & & & & & \\
\hline 2 & 0,64 & & & & & & & & & & & & \\
\hline 3 & 0,67 & 0,70 & & & & & & & & & & & \\
\hline 4 & 0,57 & 0,61 & 0,67 & & & & & & & & & & \\
\hline 5 & 0,41 & 0,46 & 0,47 & 0,46 & & & & & & & & & \\
\hline 6 & 0,31 & 0,28 & 0,32 & 0,33 & 0,29 & & & & & & & & \\
\hline 7 & 0,33 & 0,28 & 0,32 & 0,31 & 0,22 & 0,49 & & & & & & & \\
\hline 8 & 0,30 & 0,27 & 0,25 & 0,25 & 0,17 & 0,24 & 0,83 & & & & & & \\
\hline 9 & 0,19 & 0,41 & 0,23 & 0,26 & 0,26 & 0,41 & 0,43 & 0,36 & & & & & \\
\hline 10 & 0,22 & 0,18 & 0,17 & 0,21 & 0,17 & 0,20 & 0,17 & 0,19 & 0,12 & & & & \\
\hline 11 & 0,23 & 0,12 & 0,18 & 0,22 & 0,24 & 0,25 & 0,23 & 0,19 & 0,18 & 0,63 & & & \\
\hline 12 & 0,14 & 0,13 & 0,14 & 0,16 & 0,22 & 0,17 & 0,11 & 0,12 & 0,08 & 0,39 & 0,64 & & \\
\hline 13 & 0,11 & 0,14 & 0,09 & 0,13 & 0,13 & 0,03 & 0,17 & 0,09 & 0,06 & 0,22 & 0,33 & 0,50 & \\
\hline 14 & 0,19 & 0,23 & 0,24 & 0,26 & 0,22 & 0,20 & 0,15 & 0,19 & 0,15 & 0,29 & 0,27 & 0,34 & 0,32 \\
\hline
\end{tabular}

à proximidade geográfica. Dentro da região do cerrado foram encontrados, também, baixos valores de similaridade como, por exemplo, entre uma área de Nova Xavantina (MT5) e o Parque Nacional de Brasília. Em contrapartida, a área MT5 apresentou maior similaridade florística com Alter do Chão 2, Amapá e Humaitá (savanas amazônicas). Desta maneira, é

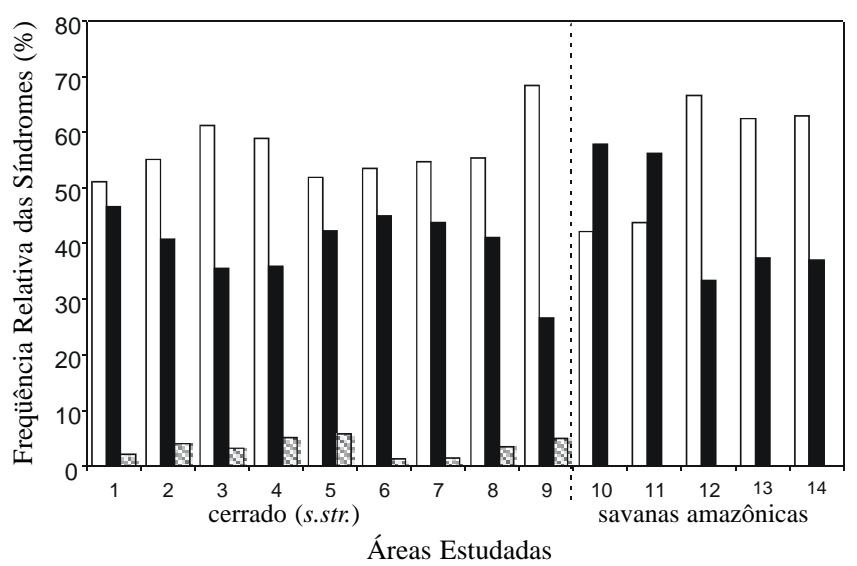

Figura 2. Proporção de síndromes de dispersão nas áreas estudadas: Cerrado sensu stricto do Brasil Central: 1-5. Nova Xavantina/Cachimbo (MT-1 a MT-5); 6. Estação Ecológica de Águas Emendadas (EEAE/DF); 7. Área de Proteção Ambiental Gama-Cabeça do Veado (APA/DF); 8. Parque Nacional de Brasília (PNB/DF) e 9. Parque Nacional de Emas (EMAS /GO). Savanas amazônicas: 10. Alter do Chão 1; 11. Alter do Chão 2; 12. Amapá; 13. Roraima e 14. Humaitá. Zoocoria $(\square)$; anemocoria (ם); autocoria $(\mathbb{Q})$. importante ressaltar que a similaridade florística está em função do número de espécies observadas em cada local. Das oito espécies que ocorreram em Roraima, seis estavam na APA (região do cerrado), porém a similaridade entre estas duas áreas foi de apenas 0,17 .

Embora os valores de similaridade entre as áreas tenham sido baixos, as proporções das síndromes (anemocoria e zoocoria) foram semelhantes entre as áreas de cada região e entre regiões.

As proporções das síndromes de dispersão encontradas no cerrado, em outros estudos, foram semelhantes às encontradas neste trabalho. Gottsberger

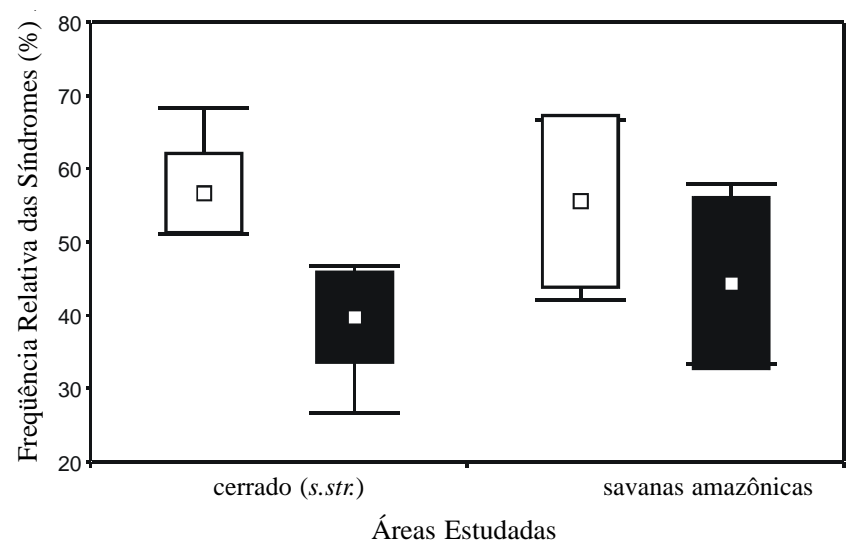

Figura 3. Frequiência relativa de zoocoria $(\square)$ e anemocoria (घ) nas savanas amazônicas $(\mathrm{n}=5)$ e cerrado sensu stricto do Brasil Central $(n=9)$. Média, média \pm DP e valores máximo e mínimo são apresentados. 
\& Silberbauer-Gottsberger (1983) encontraram, para plantas lenhosas de um cerrado em Botucatu - SP, zoocoria em $65 \%$ das espécies, anemocoria em $33 \%$ e autocoria em 2\%. Batalha \& Mantovani (2000) observaram zoocoria em $62 \%$ das espécies, anemocoria em $26 \%$ e autocoria em $12 \%$. Este valor de autocoria foi o maior registrado até o momento e deve indicar algum padrão local. Para um cerrado no Distrito Federal, Oliveira \& Moreira (1992) encontraram anemocoria em $41 \%$ das espécies.

Gentry (1982) observou que, nas florestas neotropicais, a proporção de espécies zoocóricas diminui das áreas úmidas em direção às áreas secas. Trabalhos realizados em florestas tropicais úmidas mostraram que a proporção de espécies anemocóricas foi inferior a $20 \%$ e a proporção de zoocóricas superior a $80 \%$ (Gentry 1982, Morellato \& Leitão Filho 1992, Talora \& Morellato 2000), com exceção de um estudo em floresta decídua na Venezuela (Wikander 1984), que encontrou $42 \%$ de anemocoria. Os resultados do presente trabalho mostraram que, na região do cerrado (s.str.) e das savanas amazônicas, a proporção de espécies anemocóricas foi superior a $26,7 \%$ e a de zoocóricas inferior a $68,3 \%$. Estes dados confirmam a importância das espécies anemocóricas em áreas abertas.

Todas as espécies que ocorreram nas áreas de savanas amazônicas apresentaram sementes aladas ou endozoocóricas, dispersas principalmente por aves e mamíferos, indicando que estes modos de dispersão fornecem maior sucesso na colonização de áreas distantes e/ou isoladas. Tal fato está de acordo com as idéias propostas por Kubitzki (1983), que ressaltou a importância de diásporos dispersos pelo vento e por aves entre as espécies de savanas. De acordo com Willson (1983), as espécies cujas sementes são dispersas somente após passarem pelo trato digestivo de animais (endozoocoria) alcançam maiores distâncias. Carlquist (1974) também mostrou que a endozoocoria por aves foi a síndrome mais importante em ilhas do Pacífico e Polinésia.

A autocoria é uma estratégia de dispersão raramente encontrada no cerrado (s.str.) e não foi observada em nenhuma das áreas de savanas amazônicas estudadas. Esta ausência pode estar relacionada, entre outros fatores, com: a) pequena probabilidade de se encontrar espécies autocóricas, devido ao reduzido número total de espécies nestas áreas; b) o tamanho da área amostral utilizada, que pode ter dificultado a detecção de espécies autocóricas; e c) a baixa eficiência destas espécies em se dispersar para áreas distantes ou isoladas, como é o caso das savanas amazônicas.
Estudos que enfoquem habilidade de dispersão a longa distância, ao nível específico, juntamente com maior entendimento sobre a origem das savanas amazônicas, devem esclarecer mais sobre o conjunto de espécies encontrado nesta região.

Agradecimentos - Ao Conselho Nacional de Desenvolvimento Científico e Tecnológico (CNPq) pelas bolsas de mestrado concedidas ao primeiro e quarto autores e bolsas de doutorado concedidas ao segundo e terceiro autores.

\section{Referências bibliográficas}

ÁLVARES-DA-SILVA, O. 1996. Ecologia evolutiva de um cerrado senso stricto do Parque Nacional das Emas, GO. Dissertação de mestrado, Universidade Federal de Goiás, Goiânia.

BATALHA, M.A. \& MANTOVANI, W. 2000. Reproductive phenological patterns of cerrado plant species at the Pé de Gigante Reserve (Santa Rita do Passa Quatro, SP, Brazil): a comparison between the herbaceous and woody floras. Revista Brasileira de Biologia 60:129-145.

CARLQUIST, S. 1974. Island Biology. Columbia University Press, New York.

FELFILI, J.M., SILVA-JÚNIOR, M.C., REZENDE, A.V., MACHADO, J.W.B., WALTER, B.M.T., SILVA, P.E.N. \& HAY, J.D. 1993. Análise comparativa da florística e fitossociologia da vegetação arbórea do cerrado sensu stricto na Chapada Pratinha, DF, Brasil. Acta Botanica Brasilica 6:27-46.

GENTRY, A.H. 1982. Patterns of neotropical plant species diversity. Evolution Biology 15:1-84.

GOTTSBERGER, G. \& MORAWETZ, W. 1986. Floristic, structural and phytogeographical analysis of the savannas of Humaitá (Amazonas). Flora 178:41-71.

GOTTSBERGER, G. \& SILBERBAUER-GOTTSBERGER, I. 1983. Dispersal and distribution in the cerrado vegetation of Brazil. Sonderbänd des Naturwissenschaftlichen Vereins in Hamburg 7:315-352.

HOWE, H.F. \& SMALLWOOD, J. 1982. Ecology of seed dispersal. Annual Review of Ecology and Systematics 13:201-228.

KUBITZKI, K. 1983. Dissemination biology in the savanna vegetation of Amazonia. Sonderbänd des Naturwissenschaftlichen Vereins in Hamburg 7:353-357.

MENDONÇA, R.C., FELFILI, J.M., WATER, B.M.T., SILVA JÚNIOR, M.C., REZENDE, A.V., FILGUEIRAS, T.S. \& NOGUEIRA, P.E. 1998. Flora vascular do Cerrado. In Cerrado: ambiente e flora (S.M. Sano \& S.P. Almeida, eds.). Embrapa-Cerrados, Planaltina, p.289-556.

MIRANDA, I.S. 1991. Estrutura e fenologia de uma comunidade arbórea da savana amazônica de Alter-doChão, Pará. Dissertação de mestrado, Instituto Nacional de Pesquisas da Amazônia, Manaus. 
MIRANDA, I.S. 1993. Estrutura do estrato arbóreo do cerrado amazônico de Alter do Chão, Pará, Brasil. Revista Brasileira de Botânica 16:143-150.

MORELLATO, L.P.C. \& LEITÃO FILHO, H.F. 1992. Padrões de frutificação e dispersão na serra do Japi. In História natural da serra do Japi: ecologia e preservação de uma área florestal no sudeste do Brasil (L.P.C. Morellato, org.). Editora da UNICAMP, Campinas, p.112-140.

ODUM, E.P. 1988. Ecologia. Editora Guanabara Koogan, Rio de Janeiro.

OLIVEIRA, P.E.A.M. \& MOREIRA, A.G. 1992. Anemocoria em espécies do cerrado e mata de galeria de Brasília, DF. Revista Brasileira de Botânica 15:163-174.

PRANCE, G.T. 1987. Biogeography of neotropical plants. In Biogeography and quaternary history in tropical America (T.C. Whitmore \& G.T. Prance, eds.). Oxford University Press, New York, p.46-65.

PRANCE, G.T. 1996. Islands in Amazonia. Philosophical Transactions of the Royal Society of London 351:823-833.

RATTER, J., RICHARDS, P.W., ARGENT, G. \& GIFFORD, D.R. 1973. Observations on the vegetation of northeastern Mato Grosso. I. The woody vegetation types of the Xavantina-Cachimbo expedition area. Philosophical Transactions of the Royal Society of London 266:449-492.

RATTER, J.A., BRIDGEWATER, S., ATKINSON, R. \& RIBEIRO, J.F. 1996. Analysis of the floristic composition of the Brazilian cerrado vegetation II: Comparation of the woody vegetation of 98 areas. Edinburgh Journal of Botany 53:153-180.
RATTER, J.A., BRIDGEWATER, S., RIBEIRO, J.F., DIAS, T.A.B. \& SILVA, M.R. 2000. Distribuição das espécies lenhosas da fitofisionomia Cerrado sentido restrito nos estados compreendidos pelo bioma Cerrado. Boletim do Herbário Ezechias Paulo Heringer 5:5-43.

RIBEIRO, J.F. \& WALTER, B.M.T. 1998. Fitofisionomias do bioma Cerrado. In Cerrado: ambiente e flora (S.M. Sano \& S.P. Almeida, eds.). Embrapa-Cerrados, Planaltina, p.89-166.

SANAIOTTI, T.M. 1996. The woody flora and soils of seven Brazilian amazonian dry savanna areas. $\mathrm{PhD}$ Thesis, University of Stirling, Scotland.

TALORA, D.C. \& MORELLATO, P.C. 2000. Fenologia de espécies arbóreas em floresta de planície litorânea do sudeste do Brasil. Revista Brasileira de Botânica 23:13-26.

VAN DER PIJL, L. 1982. Principles of dispersal in higher plants. $3^{\text {rd }}$ ed. Springer Verlag, New York.

VAN ROOSMALEN, M.G.M. 1985. Fruits of the guianan flora. Drukkerij Veenman B.V., Wageningen.

WIKANDER, T. 1984. Mecanismos de dispersión de diasporos de una selva decidua en Venezuela. Biotropica 16:276-283.

WILLSON, M.F. 1983. Plant reproductive ecology. Wiley Interscience Publications, New York.

ZAR, J.H. 1999. Biostatistical analysis. $4^{\text {th }}$ ed. Prentice Hall, New Jersey. 